\title{
COMPLEXITY OF CONTINUOUS SEMI-FLOWS AND RELATED DYNAMICAL PROPERTIES
}

\author{
Feng Zhang, Lian-Fa He, and Qi-Shao Lu
}

\begin{abstract}
The equicontinuity and scattering properties of continuous semi-flows are studied on a compact metric space. The main results are obtained as follows: first, the complexity function defined by the spanning set is bounded if and only if the system is equicontinuous; secondly, if a continuous semi-flow is topologically weak mixing, then it is pointwise scattering; thirdly, several equivalent conditions for the time-one map of a continuous semi-flow to be scattering are presented; Finally, for a minimal continuous map it is shown that the "non-dense" requirement is unnecessary in the definition of scattering by using open covers.
\end{abstract}

\section{Introduction}

For the need of theoretical research in topological dynamical systems, Adler, Konheim, and McAndrew presented a definition of topological entropy on a compact space by using open covers (see [1]) for continuous automorphisms, similar to that of measure entropy, and showed that it is a topologically conjugate invariant. By now, it is still one of the important invariants in dynamical systems. Since then, Bowen also gave a definition of topological entropy on a metric space for an uniformly continuous map, by spanning sets and separated sets (see [4]), and proved that the definition of topological entropy is equivalent to its definition by using open covers when the space is compact. Therefore, these have given the topological entropy a more clear and direct dynamical meaning.

From the definition of topological entropy, if a system can be approximated with an infinite precision, the dynamical meaning of topological entropy is that if the system evolves with time $n$, any orbit of length $n$ needs at least $C(n)$ orbits of length $n$ to be approximated within a predicted error, where $C(n)$ grows exponentially with $n$; that is to say, if the topological entropy of the system is $\alpha$, then $C(n) \approx e^{\alpha n}$. Therefore, when $\alpha>0$, the system is actually

Received February 20, 2006; Revised December 7, 2008.

2000 Mathematics Subject Classification. 37B05, 34C28.

Key words and phrases. continuous semi-flow, spanning set, complexity function, pointwise scattering, scattering.

Project Supported by Natural Science Foundation of P. R. China (10371030) and (A2008000132). 
complex and chaotic. People often use the topological entropy, as a criterion whether the system is chaotic. When $\alpha=0$, compared with the case of $\alpha>0$, the system is rather simple; however, from the theory and application, there still exists relatively complex and chaotic behavior. Therefore, an appealing definition of weak-chaotic system emerges (namely, its topological entropy is zero, and it is sensitive dependent) (see [8]). Furthermore, for more general research on complexity of a system, $C(n)$ is directly taken as a complexity function, and the boundedness and growth speed of $C(n)$ are used to analyse the dynamic behavior of the system. This idea was introduced by Ferenczi, first for ergodic theory (see [7]) and then for symbolic dynamic systems (see $[6]$ ). In these cases, the boundedness of the complexity function is equivalent to its equicontinuity. Recently, Blanchard and Host used open covers to definite a complexity function, for a continuous map on a compact metric space, and discussed the equicontinuous and scattering properties of the system (see [1]).

In this paper, we study the topological complexity of a continuous semi-flow. We carry to flows the notions of topological complexity defined for transformations by Blanchard, Host, and Mass and use their idea looking at spanning sets. We prove in this case the basic result that a bounded complexity for all covers is equivalent to equicontinuity, and study the opposite situation in detail, unbounded complexity for all covers.

In Section 2, the complexity function is defined by spanning sets, and the related definition of scattering is given. In Section 3, we prove that the complexity function is bounded if and only if the system is equicontinuous. In Section 4, the relation between topologically weak mixing and scattering is put forward. In Section 5, we use the related result of [3] to give several equivalent conditions for the time-one map of a continuous semi-flow to be scattering. In Section 6 , we show that, for a minimal continuous map, the requirement of "non-dense" in the definition of scattering by using open covers (see [3]) is not necessary.

\section{The definition of complexity function and scattering}

In this paper, we always suppose that $X$ is a compact metric space with a metric $d, D$ indicates the diameter of $X$. Denote $Z^{+}$as the set of positive integers, $R^{+}=[0, \infty), \varphi: R^{+} \times X \rightarrow X$ as a continuous semi-flow on $X$ ("semiflow" concisely). For $t \in R^{+}$, rewrite $\varphi_{t}=\varphi(t, \cdot): X \rightarrow X$. Let $f: X \rightarrow X$ be a continuous map. For $x \in X$ and $\epsilon>0, B(x, \epsilon)=\{y \in X \mid d(x, y)<\epsilon\}$.

Suppose that $\alpha, \beta$ are two open covers on $X$. Let $\alpha \bigvee \beta=\{U \cap V \mid U \in$ $\alpha, V \in \beta\}$, and then $\alpha \bigvee \beta$ is also an open cover on $X$. For $n \in Z^{+}$, since $f$ is a continuous map, $\bigvee_{i=0}^{n-1} f^{-i} \alpha$ is an open cover on $X . C_{o}(f, \alpha, n)$ denotes cardinality of a subcovers of $\bigvee_{i=0}^{n-1} f^{-i} \alpha$, which its cardinality is a minimum value in all subcovers, and then define a map $C_{o}(f, \alpha, \cdot): Z^{+} \rightarrow Z^{+}$, which is called a complexity function of $f$ for $\alpha$ (concisely "complexity function of $\alpha$ "). 
Definition 2.1. If for any non-dense open cover $\alpha$ of $X$ (i.e., $\alpha$ is an open cover, and for any $U \in \alpha, \bar{U} \neq X)$, the complexity function $C_{o}(f, \alpha, \cdot)$ of $\alpha$ is unbounded, then $f$ is said scattering.

Remark 2.1. According to [3], if a complexity function of a non-dense closed cover $\bar{\alpha}$ of $X$ is defined similarly, denoted by $C_{c}(f, \bar{\alpha}, \cdot)$, then $f$ is scattering if and only if for any non-dense closed cover $\bar{\alpha}$ of $X, C_{c}(f, \bar{\alpha}, \cdot)$ is unbounded.

To introduce the complexity function of a semi-flow, we need the following definition.

Definition 2.2. Let $T>0, \epsilon>0, F$ and $E$ are two subsets of $X$, if for any $x \in E$ there is $y \in F$ such that

$$
d\left(\varphi_{t}(x), \varphi_{t}(y)\right) \leq \epsilon, \forall t \in[0, T),
$$

then $F$ is a $(T, \epsilon)$ spanning set of $E$ (about $\varphi$ ).

For the continuous map $f$ and $n \in Z^{+}$and $\epsilon>0$, it is similar to define $F$ as a $(n, \epsilon)$ spanning set of $E$ (about $f$ ).

Now $C_{s}(\varphi, E, \epsilon, T)$ (resp. $\left.C_{s}(f, E, \epsilon, n)\right)$ denotes as the cardinal number of a $(T, \epsilon)$ (resp. $(n, \epsilon))$ spanning set of $E$, the cardinal number of which is minimum. So we get a map

$$
C_{s}(\varphi, E, \epsilon, \cdot): R^{+} \rightarrow Z^{+}\left(\operatorname{resp} . C_{s}(f, E, \epsilon, \cdot): Z^{+} \rightarrow Z^{+}\right),
$$

and $C_{s}(\varphi, E, \epsilon, \cdot)$ (resp. $C_{s}(f, E, \epsilon, \cdot)$ ) is called the complexity function of $\varphi$ (resp. $f$ ) about $E$ and $\epsilon$, Especially, when $E=X, C_{s}(\varphi, X, \epsilon, \cdot)$ (resp. $\left.C_{s}(f, X, \epsilon, \cdot)\right)$ is denoted as $C_{s}(\varphi, \epsilon, \cdot)$ (resp. $\left.C_{s}(f, \epsilon, \cdot)\right)$, and is concisely called the $\epsilon$-complexity function of $\varphi$ (resp. $f$ ).

Definition 2.3. If for any $\epsilon \in\left(0, \frac{D}{2}\right), C_{s}(\varphi, \epsilon, \cdot)$ is unbounded, then $\varphi$ is said scattering.

If for any $\epsilon \in\left(0, \frac{D}{2}\right), x \in X$ and $\delta>0, C_{s}(\varphi, B(x, \delta), \epsilon, \cdot)$ is unbounded, then $\varphi$ is said pointwise scattering.

If for any $\epsilon \in\left(0, \frac{D}{2}\right)$, such that $C_{s}(\varphi, \epsilon, 1)=2, C_{s}(\varphi, \epsilon, \cdot)$ is unbounded, then $\varphi$ is called 2 -scattering.

Remark 2.2. If a semi-flow is pointwise scattering, then it is scattering.

Proposition 2.1. For any open cover $\alpha$ of $X$, let $3 \epsilon(\epsilon>0)$ define the Lebesgue number of $\alpha$. If $C_{s}(f, \epsilon, \cdot)$ is bounded, then $C_{o}(f, \alpha, \cdot)$ is bounded.

Proof. When $C_{s}(f, \epsilon, \cdot)$ is bounded, we assume that $C_{s}(f, \epsilon, \cdot) \leq k(k>0)$. For any $n>0$, there is a spanning set $E=\left\{y_{1}, \ldots, y_{l}\right\}$, which is composed of $l(\leq k)$ points, such that for any $x \in X$ there exists $y_{j} \in E, j=0, \ldots, l$, satisfying $d\left(f^{i}(x), f^{i}\left(y_{j}\right)\right) \leq \epsilon, i=0, \ldots, n-1$, that is,

$$
f^{i}(x) \in B\left[f^{i}\left(y_{j}\right), \epsilon\right]=\left\{z \in X: d\left(f^{i}\left(y_{j}\right), z\right) \leq z\right\}, i=0, \ldots, n-1 .
$$

Since $3 \epsilon$ is the Lebesgue number of $\alpha$, we have an open set $U_{i, j} \in \alpha$ such that

$$
B\left[f^{i}\left(y_{j}\right), \epsilon\right] \subseteq U_{i, j} .
$$


Then we get

$$
x \in \bigcap_{i=0}^{n-1} f^{-i}\left(B\left[f^{i}\left(y_{j}\right), \epsilon\right]\right) \subseteq \bigcap_{i=0}^{n-1} f^{-i}\left(U_{i, j}\right) .
$$

This implies that $\left\{\bigcap_{i=0}^{n-1} f^{-i}\left(U_{i, j}\right), j=1, \ldots, l\right\}$ is a subcover of $\bigvee_{i=0}^{n-1} f^{-i}(\alpha)$, and $C_{o}(f, \alpha, n) \leq k$, i.e., $C_{o}(f, \alpha, \cdot)$ is bounded.

\section{Equicontinuity and boundedness of complexity functions}

In this section, we still suppose that $X$ is a compact metric space, $\varphi$ is a continuous semi-flow on $X$, and $f: X \rightarrow X$ is a continuous map.

Definition 3.1. If for any $\epsilon>0$, there is $\delta>0$ such that

$$
d(x, y)<\delta, \forall x, y \in X \Rightarrow d\left(\varphi_{t}(x), \varphi_{t}(y)\right)<\epsilon, \forall t \geq 0 .
$$

(resp. $\left.d(x, y)<\delta, \forall x, y \in X \Rightarrow d\left(f^{n}(x), f^{n}(y)\right)<\epsilon, \forall n \geq 0\right)$,

then $\varphi$ (resp. $f$ ) is said to be equicontinuous.

Proposition 3.1. $\varphi$ is equicontinuous if and only if for any $\epsilon>0 C_{s}(\varphi, \epsilon, \cdot)$ is bounded.

To prove Proposition 3.1, we need the following lemma.

Lemma 3.1. (1) $\varphi$ is equicontinuous if and only if $\varphi_{1}$ is equicontinuous.

(2) For any $\epsilon>0$, if $C_{s}(\varphi, \epsilon, \cdot)$ is bounded, then $C_{s}\left(\varphi_{1}, \epsilon, \cdot\right)$ is bounded.

Proof. From the continuity of $\varphi$ and definition, we directly get the result.

Proof of Proposition 3.1. " $\Rightarrow$ " If $\varphi$ is equicontinuous, then for any $\epsilon>0$, there is $\delta>0$ such that

$$
d(x, y)<\delta, \forall x, y \in X \Rightarrow d\left(\varphi_{t}(x), \varphi_{t}(y)\right)<\epsilon, \forall t \geq 0 .
$$

Since $X$ is compact, there exist $x_{1}, \ldots, x_{k} \in X$, such that $\bigcup_{i=1}^{k} B\left(x_{i}, \delta\right)$ covers $X$, then $\left\{x_{1}, \ldots, x_{k}\right\}$ is a $(T, \epsilon)$ spanning set of $\varphi$. Therefore $C_{s}(\varphi, \epsilon, \cdot)$ is bounded.

" $\Leftarrow "$ From the condition and Lemma 3.1(2), we know that for any $\epsilon>0$, when $C_{s}(\varphi, \epsilon, \cdot)$ is bounded, $C_{s}\left(\varphi_{1}, \epsilon, \cdot\right)$ is bounded. From Lemma 3.1(1), we only need to prove that $\varphi_{1}$ is equicontinuous.

The following idea comes from [3]. If $\varphi_{1}$ is not equicontinuous, then there is $\epsilon_{0}>0$ and a point $x_{0} \in X$, such that for any $\eta_{n}>0$ there is $y_{n} \in X$ and $k_{n} \geq 1$, satisfying $d\left(x, y_{n}\right)<\eta_{n}$, and

$$
d\left(\varphi_{1}^{k_{n}}\left(x_{0}\right), \varphi_{1}^{k_{n}}\left(y_{n}\right)\right) \geq \epsilon_{0} .
$$

Therefore we get finite points $x_{1}, \ldots, x_{k}$ of $X$, such that $\alpha=\left\{B\left(x_{1}, \frac{\epsilon_{0}}{4}\right), \ldots\right.$, $\left.B\left(x_{k}, \frac{\epsilon_{0}}{4}\right)\right\}$ is an open cover of $X$. Denote $\bar{\alpha}=\left\{\overline{B\left(x_{1}, \frac{\epsilon_{0}}{4}\right)}, \ldots, \overline{B\left(x_{k}, \frac{\epsilon_{0}}{4}\right)}\right\}$. Let $3 \epsilon$ be the Lebesgue number of $\alpha$, with $\epsilon \leq \frac{\epsilon_{0}}{6}$. Since $C_{s}\left(\varphi_{1}, \epsilon, \cdot\right)$ is bounded and Proposition 2.1, $C_{o}\left(\varphi_{1}, \alpha, \cdot\right)$ is bounded, and then $C_{o}\left(\varphi_{1}, \bar{\alpha}, \cdot\right)$ is also bounded. Furthermore, from Lemma 2.1 of [3], there is a closed cover $\left\{D_{1}, \ldots, D_{m}\right\}$ of 
$X$ satisfying $D_{i}=\bigcap_{j=0}^{\infty} \varphi_{1}^{-j}\left(\overline{B\left(x_{i, j}, \frac{\epsilon_{0}}{4}\right)}\right)\left(x_{i, j} \in\left\{x_{1}, \ldots, x_{k}\right\}\right)$. From this we know that, if $y, z \in D_{i}$, then $d\left(\varphi_{1}^{n}(y), \varphi_{1}^{n}(z)\right) \leq \frac{\epsilon_{0}}{2}<\epsilon_{0}, \forall n \in Z^{+}$. On the other hand, if $\eta_{n} \rightarrow 0$, then $y_{n} \rightarrow x_{0}$. Therefore, when $n$ is big enough, there are $y_{n}, x_{0} \in D_{i}$ (for some $i \in\{1, \ldots, m\}$ ), such that $d\left(\varphi_{1}^{k_{n}}\left(x_{0}\right), \varphi_{1}^{k_{n}}\left(y_{n}\right)\right) \geq \epsilon_{0}$. This leads to a contradiction.

\section{The relation between topologically weak mixing and scattering}

For $x \in X$, denote $O(\varphi, x)=\left\{\varphi_{t}(x) \mid t \geq 0\right\}, O(f, x)=\left\{f^{n}(x) \mid n \geq 0\right\}$.

Definition 4.1. (1) If there is $x \in X$ such that $\overline{O(\varphi, x)}=X$ (resp. $\overline{O(f, x)}=$ $X)$, then $\varphi$ (resp. $f$ ) is called topologically transitive.

(2) Let $\varphi \times \varphi($ resp. $f \times f)$ be a semi-flow on $X \times X$. If $\varphi \times \varphi($ resp. $f \times f)$ is topologically transitive, then $\varphi$ (resp. $f$ ) is called topologically weak mixing.

Remark 4.1. $\varphi$ (resp. $f$ ) is topologically transitive if and only if for any two nonempty open sets $U, V \subseteq X$, there is $t \geq 0$ (resp. $n \geq 0$ ) such that $\varphi_{t}^{-1}(U) \cap V \neq$ $\emptyset$ (resp. $\left.f^{-n}(U) \cap V \neq \emptyset\right)$.

Proposition 4.1. If $\varphi$ is topologically weak mixing, then $\varphi$ is pointwise scattering.

Proof. We claim that for any $x \in X, \delta>0$ and $N>0$, there is $T>0$ such that

$$
C_{s}(\varphi, B(x, \delta), \epsilon, T) \geq N .
$$

Since $\varphi$ is topologically weak mixing, namely $\varphi \times \varphi$ is topologically transitive, it follows from Remark 4.1 that for open sets $U_{i}, V_{i} \subseteq X,(i=1,2)$ there is $t \geq 0$ such that

$$
\left(\varphi_{t} \times \varphi_{t}\right)^{-1}\left(U_{1} \times V_{1}\right) \bigcap\left(U_{2} \times V_{2}\right) \neq \emptyset,
$$

namely, $\varphi_{t}^{-1}\left(U_{1}\right) \bigcap U_{2} \neq \emptyset, \varphi_{t}^{-1}\left(V_{1}\right) \bigcap V_{2} \neq \emptyset$.

Let $\epsilon \in\left(0, \frac{D}{2}\right)$. Then there is $\tau>0$ and open sets $U, V \subseteq X$ such that $\epsilon+2 \tau \in\left(0, \frac{D}{2}\right), \operatorname{diam}(U)<\tau, \operatorname{diam}(V)<\tau$ and $d(U, V)>2 \epsilon(d(U, V)=$ $\inf \{d(x, y) \mid x \in U, y \in V\})$. Now for any $x \in X$ and $\delta>0$, there is $t_{1} \in R^{+}$ such that

$$
\varphi_{t_{1}}^{-1}(U) \cap B(x, \delta) \neq \emptyset, \quad \varphi_{t_{1}}^{-1}(V) \cap B(x, \delta) \neq \emptyset .
$$

We choose points $x_{1}, x_{1}^{\prime} \in B(x, \delta)$ such that $\varphi_{t_{1}}\left(x_{1}\right) \in U, \varphi_{t_{1}}\left(x_{1}^{\prime}\right) \in V$. It is clear that $x_{1} \neq x_{1}^{\prime}$. Since $\varphi$ is continuous, there is a neighborhood $V_{1} \subset B(x, \delta)$ of $x_{1}^{\prime}$ such that $\varphi_{t_{1}}\left(V_{1}\right) \subseteq V$. Hence, there are $x_{2}, x_{2}^{\prime} \in V_{1}$ and $t_{2} \in R^{+}$such that

$$
\varphi_{t_{1}}\left(x_{2}\right) \in V, \varphi_{t_{1}}\left(x_{2}^{\prime}\right) \in V\left(\varphi_{t_{1}}\left(x_{2}\right) \neq \varphi_{t_{1}}\left(x_{2}^{\prime}\right)\right)
$$

and

$$
\varphi_{t_{1}+t_{2}}\left(x_{2}\right) \in V, \varphi_{t_{1}+t_{2}}\left(x_{2}^{\prime}\right) \in U
$$


As $\varphi$ is continuous, there is a neighborhood $U_{2} \subset V$ of $x_{2}^{\prime}$ such that $\varphi_{t_{1}+t_{2}}\left(U_{2}\right) \subset$ $U$. Moreover, there are $x_{3}, x_{3}^{\prime} \in U_{2}$ and $t_{3} \in R^{+}$such that $\varphi_{t_{1}}\left(x_{3}\right) \in V$,

$$
\varphi_{t_{1}+t_{2}}\left(x_{3}\right) \in U, \quad \varphi_{t_{1}+t_{2}+t_{3}}\left(x_{3}\right) \in U, \quad \varphi_{t_{1}+t_{2}+t_{3}}\left(x_{3}^{\prime}\right) \in V .
$$

We iterate this process, and get countably many points $\left\{x_{1}, x_{2}, \ldots\right\} \subset X$ and $\left\{t_{1}, t_{2}, \ldots\right\} \subset R^{+}$satisfying:

$$
\begin{gathered}
x_{1}, x_{2}, \ldots, x_{n}, \ldots \in B(x, \delta), \\
\varphi_{t_{1}}\left(x_{1}\right) \in U, \quad \varphi_{t_{1}}\left(x_{i}\right) \in V, i=2,3, \ldots, \\
\varphi_{t_{1}+t_{2}}\left(x_{2}\right) \in V, \quad \varphi_{t_{1}+t_{2}}\left(x_{i}\right) \in U, i=3,4, \ldots, \\
\varphi_{t_{1}+t_{2}+t_{3}}\left(x_{3}\right) \in U, \quad \varphi_{t_{1}+t_{2}+t_{3}}\left(x_{i}\right) \in V, i=4,5, \ldots,
\end{gathered}
$$

when $n$ is an odd number,

$$
\varphi_{t_{1}+t_{2}+\cdots+t_{n}}\left(x_{n}\right) \in U, \quad \varphi_{t_{1}+t_{2}+\cdots+t_{n}}\left(x_{i}\right) \in V, i=n+1, n+2, \ldots,
$$

when $n$ is an even number,

$$
\varphi_{t_{1}+t_{2}+\cdots+t_{n}}\left(x_{n}\right) \in V, \quad \varphi_{t_{1}+t_{2}+\cdots+t_{n}}\left(x_{i}\right) \in U, i=n+1, n+2, \ldots,
$$

Therefore, for any given $N \geq 1$, let $T=\sum_{i=1}^{N} t_{i}$. Then $C_{s}(\varphi, B(x, \delta), \epsilon, T) \geq$ $N$. In fact, let $E$ be any $(T, \epsilon)$ spanning set of $B(x, \delta)$. Then for any $y \in B(x, \delta)$ there is $z \in E$ such that

$$
d\left(\varphi_{t}(y), \varphi_{t}(z)\right) \leq \epsilon(t \in[0, T)) .
$$

Now constructing a map $\sigma: B(x, \delta) \rightarrow E$ so that $\sigma(y)=z$, and $d\left(\varphi_{t}(y), \varphi_{t}(z)\right)$ $\leq \epsilon, \forall t \in[0, T)$, When $x_{i} \neq x_{j}\left(x_{i}, x_{j} \in B(x, \delta), i, j \in\{1,2, \ldots, N\}\right)$, there is $k \in[0, T)$ such that

$$
\varphi_{k}\left(x_{i}\right) \in U, \varphi_{k}\left(x_{j}\right) \in V\left(\operatorname{or} \varphi_{k}\left(x_{i}\right) \in V, \varphi_{k}\left(x_{j}\right) \in U\right),
$$

and then $d\left(\varphi_{k}\left(x_{i}\right), \varphi_{k}\left(x_{j}\right)\right)>2 \epsilon$. Therefore, $d\left(\varphi_{k}\left(\sigma\left(x_{i}\right)\right), \varphi_{k}\left(\sigma\left(x_{j}\right)\right)\right)>0$. Accordingly $\sigma\left(x_{i}\right) \neq \sigma\left(x_{j}\right)$, so $\operatorname{Card}(E) \geq N, C_{s}(\varphi, B(x, \delta), \epsilon, T) \geq N$, namely, $\varphi$ is pointwise scattering.

Remark 4.2. From the proof of Proposition 4.1, we know that it is not necessary that $X$ is compact.

Definition 4.2. If for any $x \in X, \overline{O(\varphi, x)}=X(\operatorname{resp} . \overline{O(f, x)}=X)$, then $\varphi$ (resp. $f$ ) is called minimal.

Proposition 4.2. If $\varphi$ is minimal, then the following properties are equivalent:

(1) $\varphi$ is 2-scattering;

(2) $\varphi$ is scattering;

(3) $\varphi$ is topologically weak mixing. 
Proof. From the definitions and Proposition $4.1,(3) \Rightarrow(2) \Rightarrow(1)$ is obvious.

From [2] we know that for any minimal system $(X, \varphi)$, if it is not topologically weak mixing, then $(X, \varphi)$ has a non-trivial equicontinuous factor, and $\varphi$ is not scattering, nor 2-scattering. From these, we get $(1) \Rightarrow(3),(2) \Rightarrow(3)$. Therefore, they are equivalent.

\section{The scattering property of the time-one map of a topologically transitive semi-flow}

Denote $T(\varphi)=\{x \in X \mid \overline{O(\varphi, x)}=X\}, T(f)=\{x \in X \mid \overline{O(f, x)}=X\}$.

Lemma 5.1. Let $\varphi$ be a topologically transitive semi-flow on $X$. If for $x \in$ $T(\varphi)$, there exist a sequence of integers $\left\{n_{i}\right\}$ and an irrational number $\tau>0$ such that $\lim _{i \rightarrow \infty} \varphi_{n_{i}}(x)=\varphi_{\tau}(x)$, then $x \in T\left(\varphi_{1}\right)$.

The proof can be found in [11].

Proposition 5.1 ([11]). Let $\varphi$ be a minimal semi-flow on $X$. If $\varphi_{1}$ is not minimal, then there is an integer $k \geq 1$ such that for any minimal set $Y_{0} \subset X$ of $\varphi_{1}$, we have $\bigcup_{0 \leq t<\frac{1}{k}} Y_{t}=X$ (where $\left.Y_{t}=\varphi_{t}\left(Y_{0}\right)\right)$, and $\left\{Y_{t} \mid t \in\left[0, \frac{1}{k}\right)\right\}$ is the family of all minimal sets of $\varphi_{\frac{1}{k}}$, thus, it is also the family of all minimal sets of $\varphi_{1}$.

For a transitive semi-flow, we have the following similar result.

Proposition 5.2. Suppose that $\varphi$ is a transitive semi-flow on $X$. If $\varphi_{1}$ is not transitive, then for any $x \in T(\varphi)$, there is an integer $l \geq 1$ such that $\bigcup_{0 \leq t<\frac{1}{\tau}} Y_{t}=X\left(\right.$ where $\left.Y_{0}=\overline{O\left(\varphi_{1}, x\right)}, Y_{t}=\varphi_{t}\left(Y_{0}\right)\right)$ and $\varphi_{\frac{1}{l}}\left(Y_{t}\right)=Y_{t}(\forall t \in$ $\left.\left[0, \frac{1}{l}\right)\right)$.

Proof. For $x \in T(\varphi), Y_{0}=\overline{O\left(\varphi_{1}, x\right)}$ is a closed invariant set of $\varphi_{1}$. For $0 \leq$ $t \leq 1$, note that $Y_{t}=\varphi_{t}\left(Y_{0}\right)$, then $Y_{t}$ is also a closed invariant set of $\varphi_{1}, \varphi_{1}$ is transitive in the limitation of $Y_{t}$. Since $\overline{O(\varphi, x)}=X, \bigcup_{0 \leq t<1} Y_{t}=X$.

Note that $r=\inf \left\{t \in[0,1] \mid \varphi_{t}\left(Y_{0}\right)=Y_{0}\right\}$. Let us prove that $r>0$. Otherwise, there is a real sequence $\left\{t_{n}\right\}$ such that $t_{n} \rightarrow 0$ and $\varphi_{t_{n}}\left(Y_{0}\right)=Y_{0}$. Therefore, for any positive integers $k$ and $n$, we have $\varphi_{k t_{n}}\left(Y_{0}\right)=Y_{0}$. Since the real sequence $\left\{k t_{n} \mid k=1,2, \ldots ; n=1,2, \ldots\right\}$ is dense in $[0,1]$ for any $t \in[0,1]$, we have $\varphi_{t}\left(Y_{0}\right)=Y_{0}$. Then $X=Y_{0}=\overline{O\left(\varphi_{1}, x\right)}$ since $\varphi_{1}$ is not transitive. This is a contradiction.

Now let us prove that $r$ is a rational number. If not, it follows from $\varphi_{r}\left(Y_{0}\right)=$ $Y_{0}$ and $x \in Y_{0} \bigcap T(\varphi)$, then $\varphi_{r}(x) \in Y_{0}$. Hence there is an integer sequence $\left\{n_{i}\right\}$ such that $\lim _{i \rightarrow \infty} \varphi_{n_{i}}(x)=\varphi_{r}(x)$. From Lemma 5.1, $x \in T\left(\varphi_{1}\right)$, namely, $\varphi_{1}$ is transitive. This leads to a contradiction.

Now suppose that $r=\frac{k}{l}$ (where $k, l$ are positive integers, $l$ is coprime with $k$ and $k \leq l)$. It is claimed that $k=1$. If not, let $l=n k+m(1 \leq m<k)$, so $Y_{0}=\varphi_{1}\left(Y_{0}\right)=\varphi_{\frac{n k+m}{l}}\left(Y_{0}\right)=\varphi_{\frac{m}{l}} \circ \varphi_{\frac{k}{l}}^{n}\left(Y_{0}\right)=\varphi_{\frac{m}{l}}\left(Y_{0}\right)$. This leads to a contradiction with the definition of $r$. 
Finally, since $\bigcup_{0 \leq t<1} Y_{t}=X$ and $\varphi_{\frac{1}{l}}\left(Y_{0}\right)=Y_{0}$, it follows that $\bigcup_{0 \leq t<\frac{1}{l}} Y_{t}=$ $X$ and $\varphi_{\frac{1}{l}}\left(Y_{t}\right)=Y_{t}\left(\forall t \in\left[0, \frac{1}{l}\right)\right)$.

Proposition 5.3 ([3]). Suppose that $f: X \rightarrow X$ is a transitive continuous map. Then the following properties are equivalent:

(1) $f$ is not scattering;

(2) there exists a minimal map $g: Y \rightarrow Y$ on a compact metric space $Y$ such that $f \times g: X \times Y \rightarrow X \times Y$ is not transitive. When such a system exists, there is a minimal homeomorphism with the same property;

(3) there exists a minimal map $g: Y \rightarrow Y$ on a compact metric space $Y$ a closed invariant proper subset $J$ of $f \times g: X \times Y \rightarrow X \times Y$ and a positive integer $N$, such that $\bigcup_{0<n<N}\left(I d \times g^{n}\right) J=X \times Y$. When such a system exists, there is a minimal subshift with the same property.

Theorem 5.1. Let $\varphi$ be a transitive semi-flow on $X$. Then the following properties are equivalent:

(1) there exists $t>0$ such that $\varphi_{t}$ is not scattering;

(2) for any $t \geq 0, \varphi_{t}$ is not scattering;

(3) there exists a minimal semi-flow $\psi$ on a compact metric space $Y$, such that the semi-flow $\varphi \times \psi$ on $X \times Y$, which is defined by $\varphi_{t} \times \psi_{t}: X \times Y \rightarrow$ $X \times Y(\forall t \geq 0)$, is not transitive;

(4) there exists a minimal semi-flow $\psi$ on a compact metric space $Y$, a closed invariant proper subset $\sum$ of $\varphi \times \psi$ and a real $T>0$, such that $\bigcup_{0 \leq t<T}(I d \times$ $\left.\psi_{t}\right) \sum=X \times Y$.

To prove Theorem 5.1, we define a suspended semi-flow and related concepts first.

Suppose that $f: X \rightarrow X$ is a continuous map, for $a>0$, define an equivalent relation " $\sim$ " on $[0, a] \times X$ as follows:

$$
\left(t_{1}, x_{1}\right) \sim\left(t_{2}, x_{2}\right) \Longleftrightarrow\left\{\begin{array} { l } 
{ t _ { 1 } = t _ { 2 } , } \\
{ x _ { 1 } = x _ { 2 } ; }
\end{array} \text { or } \left\{\begin{array}{l}
t_{1}=a, \\
t_{2}=0, \\
x_{2}=f\left(x_{1}\right) .
\end{array}\right.\right.
$$

Denote $Y^{a}=[0, a] \times X / \sim$, and $\pi:[0, a] \times X \rightarrow Y^{a}$ is the quotient projection.

Now we define the semi-flow $\psi^{a}$ on $Y^{a}$ as follows:

$$
\psi^{a}(\tau,[t, x])=\left[t+\tau-n a, f^{n}(x)\right], n a \leq t+\tau \leq(n+1) a, \forall n \geq 0 .
$$

$\psi^{a}$ is called a suspended semi-flow of $f$ under $a, Y^{a}$ is called a suspended space induced by $f$ and $a$ (concisely "suspended space").

Remark 5.1. (1) Let $Y_{0}^{a}=\left\{[0, x] \in Y^{a} \mid x \in X\right\}$. Then $Y_{0}^{a}$ is an embedding of $X$ in $Y^{a}, Y_{0}^{a}$ is a closed invariant set of $\psi_{a}^{a}$ and satisfies $\psi_{a}^{a}([0, x])=$ $[0, f(x)], \forall[0, x] \in Y_{0}^{a}$. Therefore, $\left(Y_{0}^{a},\left.\psi_{a}^{a}\right|_{Y_{0}^{a}}\right)$ and $(X, f)$ can be considered as the same, and replace $Y^{a}, \psi^{a}, Y_{0}^{a}$ by $Y, \psi, Y_{0}$, respectively.

(2) $Y$ is a metric space according to [5].

(3) $f$ is minimal if and only if $\psi$ is minimal. 
Proof of Theorem 5.1. (2) $\Rightarrow$ (1) It is obvious.

$(1) \Rightarrow(3)$ Define a continuous semi-flow $\bar{\varphi}$ on $X$ by :

$$
\bar{\varphi}_{s}(x)=\varphi_{s t}(x), \forall x \in X, s \geq 0 .
$$

It is clear that $\bar{\varphi}$ is transitive, and $\bar{\varphi}_{1}$ is not scattering. Therefore, we assume that $\varphi_{1}$ is not scattering. We prove this theorem as follows:

Case 1: $\varphi_{1}$ is transitive.

From Proposition 5.3, there exists a compact metric space $Z$ and a minimal continuous map $f$ on $Z$, such that $\varphi_{1} \times f: X \times Z \rightarrow X \times Z$ is not transitive. Note that $Y$ is the suspended space induced by $f$ and number $1, \psi$ is the relative suspended semi-flow. It follows from Remark 5.1 that $\psi$ is minimal. It is claimed that $\varphi \times \psi$ is not transitive. If not, take a point $(x, y) \in T(\varphi \times \psi)$. For any $t \geq 0,\left(\varphi_{t}(x), \psi_{t}(y)\right) \in T(\varphi \times \psi)$, so assume $(x, y) \in X \times Z$. For any point $(u, v) \in X \times Z$, there exists a sequence $\left\{t_{i}\right\}$ such that $t_{i} \rightarrow \infty$ and $\left(\varphi_{t_{i}}(x), \psi_{t_{i}}(y)\right) \rightarrow(u, v)$. Denote $t_{i}=n_{i}+\tau_{i}\left(0 \leq \tau_{i}<1\right)$. Generally, we suppose that $\tau_{i} \rightarrow \tau \in[0,1], \varphi_{n_{i}}(x) \rightarrow a \in X, \psi_{n_{i}}(y)=f^{n_{i}}(y) \rightarrow b \in Z$. Since

$$
\left(\varphi_{\tau}(a), \psi_{\tau}(b)\right)=(u, v)
$$

and $b \in Z$, so when $\tau \in(0,1)$, we have $\psi_{\tau}(b) \notin Z=Y_{0}$. Taking account of $\psi_{\tau}(b)=v \in Z$, then $\tau=0$ or 1 . This shows that $\varphi_{1} \times f$ is transitive, so this is a contradiction. Therefore $\varphi \times \psi$ is not transitive.

Case 2: $\varphi_{1}$ is not transitive.

For $x \in T(\varphi)$, let $Y_{0}=\overline{O\left(\varphi_{1}, x\right)}$. From Proposition 5.2, there exists an integer $l \geq 1$, such that $X=\bigcup_{t \in\left[0, \frac{1}{\tau}\right)} Y_{t}$ (where $\left.Y_{t}=\varphi_{t}\left(Y_{0}\right)\right)$ and $\varphi_{\frac{1}{l}}\left(Y_{t}\right)=Y_{t}$. We claim that $\left.\varphi_{1}\right|_{Y_{0}}$ is not scattering. In fact, otherwise there exists a nondense closed cover $\bar{\alpha}=\left\{A_{1}, \ldots, A_{k}\right\}$ of $Y_{0}$, such that the complexity function $C_{c}\left(\varphi_{1}, Y_{0}, \bar{\alpha}, n\right)$ is unbounded. Let $A_{i}^{\prime}=\left(\left.\varphi_{\frac{1}{l}}\right|_{Y_{0}}\right)^{-1}\left(A_{i}\right)(i=1, \ldots, k), B_{i}=$ $\varphi\left(\left[0, \frac{1}{l}\right], A_{i}^{\prime}\right)$, then $\bar{\beta}=\left\{B_{1}, \ldots, B_{k}\right\}$ is a non-dense closed cover of $X$, and $C_{c}\left(\varphi_{1}, X, \bar{\beta}, n\right)=C_{c}\left(\varphi_{1}, Y_{0}, \bar{\alpha}, n\right)$. Therefore, $\varphi_{1}$ is scattering. This is a contradiction with the assumptions.

By using Proposition 5.3, then for $\left.\varphi_{1}\right|_{Y_{0}}$ there exists a minimal continuous map $f: Z \rightarrow Z$, such that $\left(\left.\varphi_{1}\right|_{Y_{0}}\right) \times f: Y_{0} \times Z \rightarrow Y_{0} \times Z$ is not transitive. Let $Y$ be a suspended space induced by $f$ and $\frac{1}{l}, \psi$ be the relative suspended semi-flow, then $\psi$ is minimal. As in the proof of case 1 , we know that, $\varphi \times \psi$ is not transitive.

Finally, suppose that $(\tilde{Y}, \tilde{\psi})$ is a natural extension of $(Y, \psi)$, i.e., $(\tilde{Y}, \tilde{\psi})$ is an inverse limit flow induced by $(Y, \psi)$. It is clear that $(\tilde{Y}, \tilde{\psi})$ meets the condition.

$(3) \Rightarrow(1)$ It is claimed that $\varphi_{1}$ is not scattering.

Case 1: $\varphi_{1}$ is not transitive.

Denote $Y_{0}=\overline{O\left(\varphi_{1}, x\right)}$ for $x \in T(\varphi)$. From Proposition 5.2, there exists an integer $l \geq 1$, such that $X=\bigcup_{t \in\left[0, \frac{1}{l}\right)} Y_{t}$ (where $Y_{t}=\varphi_{t}\left(Y_{0}\right)$ ), and $\varphi_{\frac{1}{\tau}}\left(Y_{t}\right)=$ $Y_{t}\left(\forall t \in\left[0, \frac{1}{l}\right)\right)$. Let $A_{1}=\varphi\left(\left[0, \frac{2}{3 l}\right], Y_{0}\right), A_{2}=\varphi\left(\left[0, \frac{2}{3 l}\right], Y_{\frac{1}{2 l}}\right)$. It is obvious that $\bar{\alpha}=\left\{A_{1}, A_{2}\right\}$ is the non-dense closed cover of $X$, and $\varphi_{1}^{-i}\left(A_{j}\right)=A_{j}(j=$ 
$1,2 ; i=0,1,2, \ldots)$. Therefore, the complexity function $C_{c}\left(\varphi_{1}, X, \bar{\alpha}, n\right)=2$ $(\forall n \geq 0)$. This shows that $\varphi_{1}$ is not scattering.

Case 2: $\varphi_{1}$ is transitive.

Suppose that $\psi_{1}$ is minimal. Since $\varphi \times \psi$ is not transitive, we know that $\varphi_{1} \times \psi_{1}$ is not transitive. From Proposition 5.3, $\varphi_{1}$ is not scattering.

Suppose that $\psi_{1}$ is not minimal. For any point $y \in Y$, let $Z_{0}=\overline{O\left(\psi_{1}, y\right)}$. It follows from Proposition 5.1 that there exists an integer $k \geq 1$ such that $Y=\bigcup_{0 \leq t<\frac{1}{k}} Z_{t}$ (where $\left.Z_{t}=\psi_{t}\left(Z_{0}\right)\right)$. For any $t \in\left[0, \frac{1}{k}\right), Z_{t}$ is the minimal set of $\psi_{1}$, and $Z_{t}$ is also the minimal set of $\psi_{\frac{1}{k}}$. Since $\varphi \times \psi$ is not transitive, as in the above proof, it follows that $\varphi_{1} \times\left(\left.\psi_{1}\right|_{Z_{0}}\right): X \times Z_{0} \rightarrow X \times Z_{0}$ is not transitive. Therefore, from Proposition 5.3 we know that $\varphi_{1}$ is not scattering.

$(3) \Rightarrow(2)$ For any $t>0$, let $\bar{\varphi}_{s}=\varphi_{s t}, \bar{\psi}_{s}=\psi_{s t}$. Then $\bar{\varphi}$ is the transitive semi-flow on $X, \bar{\psi}$ is the minimal semi-flow on $Y$, and $\bar{\varphi} \times \bar{\psi}$ is not transitive. Therefore, it follows from the proof of $(3) \Rightarrow(1)$ that $\bar{\varphi}_{1}=\varphi_{t}$ is not scattering.

$(3) \Rightarrow$ (4) Suppose that $U \neq \emptyset$ is an invariant non-dense open subset of $X \times Y$. Denote $\sum=\bar{U}$. The project from $U$ to $X$ is a non-empty open set, which includes a transitive point $x_{0}$ such that $\sum\left(x_{0}\right)$ is closed in $Y$, so it has a non-empty interior. Hence, there exists $T>0$ such that $\bigcup_{t \in[0, T)} \psi_{t}\left(\sum\left(x_{0}\right)\right)=Y$. Denote $K=\bigcup_{t \in[0, T)}\left(I d \times \psi_{t}\right) \sum$, then $K\left(x_{0}\right)=Y$. Since $x_{0}$ is a transitive point, and $Y$ is minimal for any $t_{0} \in R^{+}$, and $\sum\left(\varphi_{t_{0}}\left(x_{0}\right)\right)$ includes almost periodic points, it follows that $\bigcup_{t \in[0, T)} \psi_{t}\left(\sum\left(\varphi_{t_{0}}\left(x_{0}\right)\right)\right)=Y$, namely, $K\left(\varphi_{t_{0}}\left(x_{0}\right)\right)=Y$. For any $x \in X$, since there exists $\delta>0$ such that $d\left(\varphi_{t_{n}}\left(x_{0}\right), x\right)<\delta, d\left(K\left(\varphi_{t_{n}}\left(x_{0}\right)\right), K(x)\right)<\frac{1}{n}$ we have $d(Y, K(x))<\frac{1}{n}$ for any $n$. Therefore, we have $K(x)=Y, \forall x \in X$. Then $K=X \times Y$.

$(4) \Rightarrow(3)$ It follows from the Baire theorem (a complete metric space is second category) that the interior of $\sum$ is a non-empty and non-dense invariant set, so $\varphi \times \psi$ is not transitive. Taking account of the natural extension of $(Y, \psi)$, so we get the minimal flow having this property.

It follows from Theorem 5.1 that we have the following corollary.

Corollary 5.1. Let $\varphi$ be a transitive semi-flow on $X$. Then the following properties are equivalent:

(1) there exists $t>0$, such that $\varphi_{t}$ is scattering;

(2) for any $t>0, \varphi_{t}$ is scattering;

(3) for any minimal semi-flow $\psi, \varphi \times \psi$ is transitive.

Combining the above results with the proof of $(3) \Rightarrow(1)$ in Theorem 5.1, we have the following relation between scattering and transitivity:

Corollary 5.2. Let $\varphi$ be a transitive semi-flow on $X$. Then we have

(1) if $\varphi_{1}$ is not transitive, then for any $t>0, \varphi_{t}$ is not scattering.

(2) if $\varphi_{1}$ is scattering for any $t>0, \varphi_{t}$ is transitive.

Remark 5.2. It follows from Theorem 5.1 that the result still holds after parametrization of time $t$. 


\section{The definition of scattering on a continuous map}

In the writing of this paper, we discussed with F. Blanchard, the author of [3], about the "non-dense" limitation question in the definition of scattering for a continuous map. Generally, the "non-dense" requirement is necessary, but under which situation does it become unnecessary? Therefore, F. Blanchard brought the following question: when the system is minimal, is the "non-dense" the requirement necessary? The aim of this section is to give a positive answer to it.

Theorem 6.1. Suppose that $X$ is a compact metric space, and $f: X \rightarrow X$ is a continuous map. If $f$ is minimal, then the following properties are equivalent:

(1) $(X, f)$ is scattering;

(2) for an open cover $\alpha=\left\{A_{1}, A_{2}\right\}$, which is composed of any two non-dense open sets, $C_{o}(f, \alpha, \cdot)$ is unbounded;

(3) for an open cover $\beta=\left\{D_{1}, D_{2}\right\}$, which is composed of any two open sets, $C_{o}(f, \beta, \cdot)$ is unbounded.

Proof. It follows from [10] that (1) is equivalent to (2).

$(3) \Rightarrow(2)$ It is obvious.

$(2) \Rightarrow(3)$ Assume that there exists an open cover $\beta=\left\{D_{1}, D_{2}\right\}$, which is composed of two open sets, such that $C_{o}(f, \beta, \cdot) \leq M$ (where $M>0$ ). If the elements of $\beta$ are non-dense, obviously the claim holds. In what follows, we assume that $\bar{D}_{1}=X, D_{2}$ is non-dense (if both $\bar{D}_{1}=X$ and $\bar{D}_{2}=X$, the proof is similar). For any nonempty open sets $U, V$ and $V \subset U$, since $f$ is minimal, for any $x \in \bar{U}$ there exists $k$ such that $f^{k}(x) \in V$. Taking account of $f$ is continuous, there exists a neighborhood $V_{x}$ of $x$ such that $f^{k}\left(V_{x}\right) \subseteq V$. Since $\bar{U}$ is a compact set, we have a finite open cover $\left\{V_{1}, \ldots, V_{m}\right\}$ and $l_{1} \in Z^{+}$, to ensure that for any $x \in U$ there exists $k \in\left\{0, \ldots, l_{1}\right\}$ satisfying $f^{k}(x) \in V \subset U$. Therefore, for any $n \in Z^{+}$there exists $k_{n} \in\left\{n, n+1, \ldots, n+l_{1}\right\}$, satisfying $f^{k_{n}}(x) \in U$. It can be proved by induction. When $n=0$, it is obvious. Assuming that it holds for $n=m$, we discuss the condition for $n=m+1$. When $k_{m} \geq m+1$, it is obvious. When $k_{m}=m$, since $f^{k_{m}}(x) \in V$, there exists $t \in$ $\left\{0, \ldots, l_{1}\right\}$ such that $f^{k_{m}+t}(x) \in V \subset U$. Due to $k_{m}+t \in\left\{m+1, \ldots, m+1+l_{1}\right\}$, so it holds for $n=m+1$. For $X$ is a compact space, there exists a finite open cover $E=\left\{U_{1}, \ldots, U_{N}\right\}$ and $L$ such that for any $x \in U_{i}, i \in\{1, \ldots, N\}$ and any $n \in Z^{+}$, there exists $s \in\{n, \ldots, n+l\}$ such that $f^{s}(x) \in U_{i}$, and then $C_{o}(f, E, \cdot) \leq M N^{L}$, this is contradictory with (1). The theorem is proved completely.

\section{References}

[1] R. L. Adler, A. G. Konheim, and M. H. McAndrew, Topological entropy, Trans. Amer. Math. Soc. 114 (1965), 309-319.

[2] J. Auslander, Minimal Flows and Their Extensions, North-Holland Mathematics Studies, 153. North-Holland Publishing Co., Amsterdam, 1988. 
[3] F. Blanchard, B. Host, and A. Maass, Topological complexity, Ergodic Theory Dynam. Systems 20 (2000), no. 3, 641-662.

[4] R. Bowen, Entropy for group endomorphisms and homogeneous spaces, Trans. Amer. Math. Soc. 153 (1971), 401-414.

[5] R. Bowen and P. Walters, Expansive one-parameter flows, J. Differential Equations 12 (1972), 180-193.

[6] S. Ferenczi, Complexity of sequences and dynamical systems, Discrete Math. 206 (1999), no. $1-3,145-154$

[7] _ Measure-theoretic complexity of ergodic systems, Israel J. Math. 100 (1997), 189-207.

[8] S. Galatolo, Global and local complexity in weakly chaotic dynamical systems, Discrete Contin. Dyn. Syst. 9 (2003), no. 6, 1607-1624.

[9] L. F. He, S. H. Fu, and X. H. Yan, Some dynamical properties of the minimal continuous semi-flows, Indian J. Pure Appl. Math. 36 (2005), no. 4, 189-201.

[10] W. Huang and X. D. Ye, Topological complexity, return times and weak disjointness, Ergodic Theory Dynam. Systems 24 (2004), no. 3, 825-846.

[11] V. V. Nemiskii and V. V. Stepanov, Qualitative Theory of Differential Equations, Princeton Mathematical Series, No. 22 Princeton University Press, Princeton, N. J. 1960.

[12] K. Petersen, Disjointness and weak mixing of minimal sets, Proc. Amer. Math. Soc. 24 (1970), 278-280.

FENG Zhang

College of Mathematics and Information Science

Hebei Normal University

Shijiazhuang, 050016, P. R. China

AND

SCHOOL OF SCIENCE

Beijing University of Aeronautics and Astronautics

Beijing 100083, P. R. China

E-mail address: zhangflunwen@163.com

LiAn-FA He

College of Mathematics and Information Science

Hebei NoRmal UNIVERSITY

ShiJiazhuang, 050016, P. R. China

QI-Shao Lu

SCHOOl OF SCIENCE

Beijing University of Aeronautics and Astronautics

BeiJing 100083, P. R. China 